\title{
Typen und Strategien von Täterinnen bei sexuellem Kindesmissbrauch
}

\author{
Eine qualitative Inhaltsanalyse der Berichte von betroffenen Personen und Zeug:innen
}

\author{
Tanita Gebhardt ${ }^{1}$ Peer Briken ${ }^{1}$ S Safiye Tozdan ${ }^{1}$ Johanna Schröder ${ }^{1}$ \\ Eingegangen: 13. Juli 2021 / Angenommen: 25. Oktober 2021 / Online publiziert: 7. Dezember 2021 \\ (c) Der/die Autor(en) 2021
}

\section{Zusammenfassung}

Hintergrund Bei sexuellem Kindesmissbrauch wird in der Regel an einen männlichen Täter gedacht. Frauen, die Kinder sexuell missbrauchen, stellen in unserer Gesellschaft immer noch ein Tabuthema dar. Täter:innentypologien können ein wichtiges Hilfsmittel darstellen, um zu verbesserten Interventionsmaßnahmen, Rehabilitationsleistungen sowie zur Verbrechensprävention beizutragen. Bisherige Typologien zu weiblichen Tätern im Kontext sexuellen Kindesmissbrauchs konzentrieren sich entweder auf Persönlichkeitsmerkmale oder Verhaltensweisen. Die vorliegende Analyse vereint beide Aspekte und versucht somit, bisherige Forschung zu ergänzen.

Methodik Zur Entwicklung einer Täterinnentypologie wurden 30 schriftliche Berichte und transkribierte Anhörungen von weiblichen sowie männlichen Betroffenen und Zeug:innen mittels qualitativer Inhaltsanalyse ausgewertet.

Ergebnisse Es wurden 4 Täterinnentypen und ihre Strategien herausgearbeitet: die sadistische Täterin, die vermittelnde Täterin, die parentifizierende Täterin und die instruierende Täterin.

Schlussfolgerungen Eine Wissensvermittlung hinsichtlich der Täterinnentypen an verschiedene Institutionen der Gesellschaft könnte die Aufklärungsarbeit bezüglich weiblicher Täterinnen im Kontext sexuellen Kindesmissbrauchs intensivieren.

Schlüsselwörter Kindesmisshandlung · Gesellschaftliches Tabu · Weibliche Täterschaft · Täterinnentypologie · Kriminalprävention

\section{Types and strategies of female child sexual offenders}

A qualitative content analysis of reports of affected persons and contemporary witnesses

\begin{abstract}
Background Child sexual abuse implies the image of a male perpetrator. Women who sexually abuse children are still a taboo subject in our society. Perpetrator typologies can be an important tool to contribute to improved intervention measures, rehabilitation services and crime prevention. Previous typologies on female perpetrators in the context of child sexual abuse have focused on either personality traits or behaviors. The present analysis combines both aspects and thus attempts to complement previous research.
\end{abstract}

S. Tozdan und J. Schröder teilen sich die Letztautorinnenschaft.

Tanita Gebhardt, M.A. Krim.

tanita@tanitagebhardt.de

1 Institut für Sexualforschung, Sexualmedizin und Forensische

Psychiatrie, Universitätsklinikum Hamburg-Eppendorf,

Hamburg, Deutschland 
Methods In order to develop a typology of female perpetrators, 30 written reports and transcribed hearings of female as well as male victims and eyewitnesses were evaluated using qualitative content analysis.

Results A total of four different types of female child sexual offenders and their strategies could be identified. These are the sadistic perpetrator, the mediating perpetrator, the parentifying perpetrator and the instructing perpetrator.

Conclusion A transfer of knowledge regarding the types of female perpetrators to different institutions of society could intensify the clarificational work regarding female perpetrators in the context of child sexual abuse.

Keywords Child abuse $\cdot$ Social taboo $\cdot$ Female perpetrators $\cdot$ Perpetrator typology $\cdot$ Crime prevention

\section{Einleitung}

Sexueller Kindesmissbrauch durch Frauen ist ein tabuisiertes Thema, zu dem im Gegensatz zu männlicher Täterschaft nur wenig Forschung existiert (PhD Tozdan et al. 2019). Ein gesellschaftlich verankertes Stereotyp, welches der Frau (sexuelle) Aggression abspricht und Macht und aggressives Begehen an den Mann delegiert, führt dazu, dass Betroffene nicht ernst genommen sowie Täterinnen nicht gesehen werden (Motz 2014; Arzt et al. 2018). Dies betrifft auch professionelle Fachkräfte (Dr. Denov 2003).

Täterinnentypologien können für Fachkräfte in der Präventionsarbeit und in der Behandlung von Frauen mit einem sexuellen Interesse an Kindern, die ein Risiko für sexuelle Übergriffe auf Kinder aufweisen, ein wichtiges Hilfsmittel darstellen. Sie gelten als relevant bei der Entwicklung von Interventionsmaßnahmen und Rehabilitationsleistungen sowie als Werkzeug der Verbrechensprävention (Sandler und Freeman 2007). Betrachtet man Typologien weiblicher Sexualstraftäterinnen werden diese zum einen nach ihren Persönlichkeitsmerkmalen unterschieden, zum anderen konzentrieren sie sich auf Verhaltensweisen der Täterinnen (Niemeczek 2014). Die Typologien beschreiben oftmals Frauen, die in starker Abhängigkeit und unter Zwang eines Mannes missbrauchen (Matthews et al. 1991; Enders 1995; Saradjian und Hanks 1996; Prof. Dr. Kavemann und Braun 2002), oder auch Frauen, die durch ihre eigenen Missbrauchserfahrungen, vorrangig in der Kindheit und z. T. auch intergenerational, vorbelastet sind (Matthews et al. 1991; Saradijan und Hanks 1996; Prof. Dr. Kavemann und Braun 2002). Weiterhin werden Frauen beschrieben, die Schwierigkeiten haben, ihr Verhalten selbst als kriminell zu verstehen, da ihre Intention nicht mit Boshaftigkeit behaftet sei und die sexuellen Interaktionen mit heranwachsenden Jungen von der Frau als positiv und vorteilhaft für den Jungen gewertet werden (Matthews et al. 1991). Dieser Typus wird von weiteren Täterinnentypologien aufgegriffen (Syed und Williams 1996; Prof. Dr. Kavemann und Braun 2002). Psychische Krankheitsbilder sowie Suchterkrankungen, welche Einfluss auf das Missbrauchsverhalten nehmen, werden ebenfalls häufig genannt (Johansson-Love und Fremouw 2006).
Diese bisherigen Täterinnentypologien haben gemeinsam, dass sie die Frau als Täterin zum größten Teil als sehr passiv, vorbelastet oder nur vermindert zurechnungsfähig darstellen. Es besteht Grund zur Annahme, dass sie aufgrund dessen weniger Verantwortung für den Missbrauch trägt und ein Phänomen wie z.B. das Vorliegen eines (sexuellen) Sadismus als eher unwahrscheinlich erachtet wird. Ein weiterer Grund für diese Annahme könnte in der Schwierigkeit der Datenerhebung liegen. Täterinnen sexuellen Kindesmissbrauchs bewegen sich zum größten Teil im Dunkelfeld, wodurch es schwierig ist, aussagekräftige und verwertbare Daten zu erheben.

Sexueller Missbrauch setzt in der Regel ein methodisches, strukturiertes Vorgehen voraus, in denen Täter:innen eine aktive Rolle spielen müssen, um ihr Ziel zu erreichen (Bullens 1995). Ziel dieser Strategien ist einerseits, den Zugang zu Betroffenen zu ermöglichen, diese gefügig $\mathrm{zu}$ machen und jeglichen existierenden Widerstand aufzulösen sowie durch Schuldverschiebungen, Drohungen und Geheimhaltung als Täter:in unentdeckt zu bleiben (Dr. Heiliger 2001). In der vorliegenden Studie soll untersucht werden, welche Rolle Frauen im Kontext sexuellen Kindesmissbrauchs spielen, und welche Strategien sie dabei verfolgen. Gegenstand der Datenanalysen sind schriftliche Berichte und transkribierte Interviews von Betroffenen, die sexualisierte Gewalt durch eine oder mehrere Frauen erlebt haben.

\section{Methodik}

\section{Studiendesign und -material}

Im Jahr 2016 berief der Unabhängige Beauftragte für Fragen des sexuellen Kindesmissbrauchs (UBSKM), basierend auf einem Beschluss des Deutschen Bundestags, die Unabhängige Kommission zur Aufarbeitung sexuellen Kindesmissbrauchs (UKASK). Der Auftrag dieser Kommission sollte es sein, Ursachen, Bedingungen und Folgen von sexuellem Kindesmissbrauch in Deutschland zu untersuchen, gemeinsam mit Betroffenen über das Ausmaß von sexuellem Kindesmissbrauch zu informieren und für das Thema zu sensibilisieren (Unabhängige Kommission zur Auf- 
arbeitung sexuellen Kindesmissbrauchs 2019). Durch die UKASK wurden transkribierte Anhörungen sowie schriftliche Berichte Betroffener und Zeug:innen zur Verfügung gestellt, in denen die Täterschaft einer Frau deutlich wurde. Sämtliche Angaben, welche Rückschluss auf betroffene Personen oder Orte erlaubten, wurden von Mitarbeiter:innen der UKASK zensiert und nach Absprache mit Betroffenen und Zeug:innen und deren Zustimmung doppelt pseudonymisiert.

\section{Datenanalyse}

Die Software ATLAS.ti (Scientific Software Development $\mathrm{GmbH}$ ) (2018) wurde genutzt, um die Dokumente im Sinne der qualitativen Inhaltanalyse nach Mayring (2010) auszuwerten. In einem ersten Durchlauf wurden relevant erscheinende Textstellen bezüglich der Rolle der Täterin markiert, welche Aussagen über ihre Persönlichkeitsmerkmale beinhalteten. Diese Texteinheiten wurden im weiteren Verlauf auf ein einheitliches Sprachniveau gehoben und zu Einheiten zusammengefasst, um in einem darauffolgenden Schritt gleiche und/oder ähnliche Generalisierungen in einer $\mathrm{zu}$ vereinen. Auf dieser Grundlage entstand ein induktives Kategoriensystem, welches die verschiedenen Täterinnentypen repräsentiert. In einem weiteren Schritt wurde dieses Kategoriensystem genutzt, um die den Typen zugeordneten Dokumente hinsichtlich spezifischer Täterinnenstrategien zu analysieren. Das zweistufige Verfahren ermöglichte das Erstellen einer Täterinnentypologie, die Persönlichkeitsmerkmale sowie Handlungsweisen vereint.

\section{Ergebnisse}

\section{Stichprobenbeschreibung}

Die 40 von den Mitarbeitenden der UKASK übermittelten Dokumente wurden auf ihren Informationsgehalt hinsichtlich der vorliegenden Fragestellung überprüft. Hiervon enthielten 30 Dokumente genügend Informationen, um die Fragestellungen zu beantworten, wohingegen 10 Dokumen-

Tab. 1 Stichprobenzusammensetzung $(n=30)$

\begin{tabular}{lll}
\hline Analysiertes Material & & \\
Anhörung/Interview $(n, \%)$ & 18 & 60,0 \\
Schriftlicher Bericht $(n, \%)$ & 12 & 40,0 \\
Quelle des Berichts & & \\
Primär $(n, \%)$ & 28 & 93,3 \\
Sekundär $(n, \%)$ & 2 & 6,7 \\
Geschlecht der Berichtenden & & \\
Männlich $(n, \%)$ & 11 & 36,7 \\
Weiblich $(n, \%)$ & 19 & 63,3 \\
\hline
\end{tabular}

te aufgrund ihres mangelnden Informationsgehalts von der Analyse ausgeschlossen wurden .

Die Stichprobe beinhaltet 18 transkribierte Anhörungen und 12 schriftliche Berichte von Betroffenen und Zeug:innen, wobei es sich bei beiden Zeug:innen um ein Elternteil einer betroffenen Person handelt. Insgesamt setzen sich die untersuchten Fälle aus 11 männlichen Personen und 19 weiblichen Personen zusammen (Tab. 1). Die von ihnen beschriebenen Täterinnen stammten $\mathrm{zu}$ 58,3\% aus dem familiären Umfeld, wobei die leibliche Mutter am häufigsten genannt wurde $(32,6 \%)$, gefolgt von der Großmutter $(6,9 \%)$. Am zweithäufigsten ließen sich die Täterinnen in das nähere soziale Umfeld einordnen, wo sie einen Prozentsatz von $25 \%$ ausmachten. Hier wurden v.a. Frauen im Rahmen von Institutionen wie dem Kindergarten sowie katholischer und evangelischer Einrichtungen genannt. Das bedeutet, dass insgesamt 83,3\% der Täterinnen dem engeren sozialen Umfeld der Betroffenen zuzuordnen waren. In 16,7\% der Fälle handelte es sich um Fremdtäterinnen.

\section{Täterinnentypen}

In der Datenanalyse bildeten sich 4 verschiedene Typen weiblicher Täterinnen und ihre Strategien im Kontext des sexuellen Kindesmissbrauchs heraus - die sadistische Täterin, die vermittelnde Täterin, die parentifizierende Täterin und die instruierende Täterin. Die sadistische Täterin sowie die parentifizierende Täterin sind mit 12 Fällen am häufigsten vertreten, gefolgt von einer Mischform der vermittelnden Täterin und der sadistischen Täterin mit insgesamt 8 Fällen. Es folgt der Typus der vermittelnden Täterin mit 7 Fällen. Die instruierende Täterin sowie eine Mischform der lehrenden Täterin und der vermittelnden Täterin treten jeweils ein einziges Mal auf.

In der folgenden Ergebnisdarstellung werden die einzelnen Täterinnentypen systematisch beschrieben und mit Beispielzitaten belegt. In 24 von 30 Fällen wurde einer $\mathrm{Zi}$ tatfreigabe zugestimmt, während 6 Fälle diese verweigert haben. Hierbei handelte es sich um 2 Fälle der parentifizierenden Täterin, 2 Fälle der vermittelnden Täterin sowie einen Fall der sadistischen Täterin und einen Fall der Mischform der sadistischen und vermittelnden Täterin.

\section{Die sadistische Täterin}

Berichtende, die diesen Typ Täterin beschreiben, betonen ein enormes Ausmaß an Gewalt. Die (sexuelle) Befriedigung durch und am beobachtbaren Leid des betroffenen Kindes ist als zentral für den Typus der sadistischen Täterin anzusehen. Die Hilflosigkeit sowie das Schmerzempfinden des Kindes erregen die sadistische Täterin, „... ob es nun anal war oder anders irgendwie, auch unter Schläge, dass sie mich geschlagen hat und sie sich dabei selbstbefriedigt 
hat [...] Umso mehr ich gelitten hab und gezeigt hab, wie ... wie weh mir das tut und ... und ich geweint hab, umso mehr hat sie ihre Freude gehabt". Die Handlungen der sadistischen Täterin sind von extremer Gewalt geprägt. Die sexualisierte, körperliche und psychische Gewalt der Täterinnen umfasst das Deponieren spitzer Gegenstände im Essen der Betroffenen, Fesselungen, Strangulation, Abschnürung von Körperteilen, Nahrungsmittelentzug und Belohnungsfüttern, vaginale und anale Penetrationen mit Gegenständen und Verstümmelung durch das Abtrennen der Schamlippen. Auf Grundlage der Berichte betroffener Personen wird die Ausübung von Macht als zentrales Motiv deutlich.

Das hohe Ausmaß an Gewalt spiegelt sich ebenfalls in den Täterinnenstrategien der sadistischen Täterin wider. Die sadistische Täterin scheint sich des Kindes Unwissenheit und Unsicherheit zunutze zu machen, um es für ihre Zwecke zu manipulieren. So führt das Darstellen des Kindes als Lügner:in dazu, dass dieses den eigenen Wahrnehmungen nicht mehr vertraut, ,es wurde systematisch verbreitet und natürlich mir auch selber eingeimpft, dass ich lüge, [...] egal, was ich sage. [...], weil ich selber ja dann auch gar nicht wusste, stimmt das oder nicht". Das Zuweisen von Schuld suggeriert dem Kind zudem, dass es den Missbrauch verdient habe, ,wir haben von ihr als grundlegende Botschaft vermittelt bekommen, dass wir triebhaft und verdorben sind und Schuld an allem". Um ein mögliches Offenbaren des Missbrauchsgeschehens an Dritte zu verhindern, bedroht sowie bestraft die sadistische Täterin die Betroffenen, ,, wenn man dann geschrien hat, [...] und dann ist das Wasser in die Nase reingetrichtert worden ". Weiterhin verunsichert die Sadistin die Betroffenen durch Schikane, „,alles wurde kommentiert und über alles wurde sich lustig gemacht. [...] Alles, was ich gemacht hab, war falsch. Den ganzen Tag. Ich wusste schon gar nicht mehr, was ich überhaupt noch machen sollte". Die daraus resultierende Unsicherheit führt beim Kind dazu, dass die Empfänglichkeit für weitere Strategien steigt und die sadistische Täterin den Missbrauch somit beliebig lange fortsetzen kann.

\section{Die vermittelnde Täterin}

Bei der vermittelnden Täterin handelt es sich um einen Typ Täterin, der betroffene Kinder Dritten zuführt, damit diese die Kinder sexuell missbrauchen können. Dabei kann es sich um zahlende Kund:innen handeln, andererseits aber auch um Personen aus dem familiären und sozialen Nahraum. Die vermittelnde Täterin verschafft sich durch die Übergabe einen Vorteil. Dieser Vorteil kann finanzieller Natur sein, in dem sie Geld von Kund:innen einfordert, oder persönlicher Natur, da sie so ihre Position in organisierten Strukturen erfüllt und damit sichert.

Ein anderes Motiv stellt das Umgehen des eigenen sexuellen Missbrauchs dar, sodass stattdessen der sexuelle
Missbrauch einer anderen Person bzw. des (teilweise eigenen) Kindes in Kauf genommen wird, ,meine Mutter hat mich als Kleinkind als Schutz vor ihrem alkoholisierten Ehemann mit ins Bett genommen. Ich kann mich an Situationen erinnern, wo ich von einer großen Hand, die ich zwischen meinem Schritt spürte, die meinem Vater gehörte, wach wur$d e$ “. Die vermittelnde Täterin wird zudem bei der Übergabe der betroffenen Kinder aktiv und überwacht den sexuellen Missbrauch. Hierbei spricht sie stellvertretend den Willen und Einverständnis des Kindes aus, ,einmal kam die Mutter dazu. Da war sie mit dabei. Und dann hieß das, dass wir das eben wollten ". Sollte das Kind sich gegen die Übergabe wehren, wird diese oft gewaltsam erzwungen. Auch das Auffordern Dritter zum sexuellen Missbrauch lässt sich als für den Typus charakteristisch feststellen. Als Bindeglied zwischen Betroffenen und Täter:innen nutzt die vermittelnde Täterin das Vortäuschen von Fürsorglichkeit gegenüber den Betroffenen.

Wie bei den Strategien der sadistischen Täterin ist auch bei der vermittelnden Täterin eine Schuldzuweisung gegenüber Betroffenen festzustellen, ,meine Mutter sagte oft, dass ich jetzt Schuld bin, dass der Familienfrieden gestört ist". Des Weiteren relativiert sie den Missbrauch. Dies vermittelt den Betroffenen das Gefühl, dass ihr Empfinden des Missbrauchs zu drastisch sei, und wertet somit ihr empfundenes Leid ab, ,der *(Täter) hat sich doch entschuldigt, und jetzt ist doch gut ". ${ }^{1}$ Die Rolle der vermittelnden Täterin im Missbrauchskontext wird hierdurch ebenfalls verharmlost. Die vermittelnde Täterin schafft zudem Familiarität, indem sie Betroffene dazu auffordert, Täter so anzusprechen, als würden sie in einem Verwandtschaftsverhältnis zu diesen stehen. Durch Kosenamen, welche das Kind für gewöhnlich aus seinem familiären Umfeld kennt, schafft sie eine Vertrauensbasis zwischen Kind und Täter:in. Dies erleichtert ihr die Vermittlung des Kindes. Bei der vermittelnden Täterin ist eine Vernetzung von Täter:innen zu erkennen, welche verhindert, dass Betroffene dem Missbrauch entfliehen können, sowie die Täter:innen schützt, ,und jeder wusste, wenn ich rumlaufe, wer das ist, und dass man mit mir alles machen kann. Und die Nachbarsdörfer alle haben Bescheid gewusst".

\section{Die parentifizierende Täterin}

Auffällig an der parentifizierenden Täterin als Typus sind die sexuelle Desensibilisierung des Kindes durch Gespräche sowie das Integrieren der sexuellen Übergriffe in alltägliche Situationen. Dies suggeriert dem Kind, dass der

\footnotetext{
1 *(Täter) übernommen aus zur Verfügung gestellten Transkripten der Unabhängigen Kommission zur Aufklärung Sexuellen Kindesmissbrauchs, da Originalzitat - Platzhalter für anonymisierten Namen des Täters
} 
sexuelle Missbrauch als etwas Normales zu betrachten ist, „es sei toll, wenn da Luft drankäme, und so bammelte alles immer schön herum, stell dich nicht an, ist doch ganz natürlich!'“. Beispiele hierfür sind das unerwartete in den Schritt des Kindes fassen und dabei lachen, das Anprobieren von Kleidern und Einholen der Meinung des betroffenen Kindes sowie das Mitteilen von sexuellen Wünschen, Fantasien und Einzelheiten des eigenen Sexlebens. So erzählt die parentifizierende Täterin dem Kind beispielsweise, an welchen Orten sie bereits Sex hatte, Sex haben will, oder für welche Person sie Gefühle empfindet.

Das Kind fungiert für die parentifizierende Täterin als (sexueller) Partnerersatz. Sie sieht im Kind eine:n Verbündete:n. Dabei muss die parentifizierende Täterin nicht zwingend die leibliche Mutter des Kindes sein. So fand sich dieser Täterinnentyp z.B. ebenfalls bei sexuellem Missbrauch durch die Lebensgefährtin des Vaters oder einer Frau, die den Betroffenen als Babysitter beanspruchte. In beiden Fällen missbrauchten die Frauen ihre Autoritätsposition, um das Kind zu befehligen. Auch im Erwachsenenalter demonstriert die parentifizierende Täterin ihren Anspruch gegenüber Partner:innen der Betroffenen, ,meine Frau sitzt da, ich sitze mit der Mutter da auf der Couch und da fasst sie mir so auf den Schenkel. So. Und zwar nicht so, sondern schon in der Zone, wo es unanständig ist, ja? Und ... und schaut dabei meine Frau richtig provozierend an". Ebenfalls lassen sich generationsübergreifende sexuelle Grenzüberschreitungen feststellen, sodass auch Kinder der Betroffenen von der Täterin bedrängt wurden.

Die führende Strategie der parentifizierenden Täterin besteht darin, ihre sexuelle Übergriffigkeit als etwas zu präsentieren, das von dem betroffenen Kind als normal und alltäglich angesehen werden soll. Das führt dazu, dass dieses die sexuellen Übergriffe nicht als solche wertet, sich eine Gewöhnung einstellt und die Täterin ihren Missbrauch unter dem Deckmantel des Alltäglichen fortsetzen kann, „die lief ja ewig und drei Tage lang vor mir in der Unterwäsche rum. Also das war auch so ganz selbstverständlich. Also da hast du nie drüber nachgedacht, also was ist mit meiner Mutter los, ja?".

Da das betroffene Kind für sie einen (sexuellen) Partnerersatz darstellt, werden auch Konflikte mit dem Kindsvater vor bzw. mit dem Kind thematisiert. Somit wird der andere Elternteil diffamiert, und die parentifizierende Täterin versucht, eine Art Bündnis mit dem Kind zu schlieBen, wodurch eine starke emotionale Verstrickung entsteht. Weiterhin desensibilisiert sie das Kind, indem sie sexuelle Themen anspricht und diskutiert. Es kommt insbesondere zu sexuell konnotierten Bemerkungen bezüglich des kindlichen Körpers. Lässt das Kind den Missbrauch der parentifizierenden Täterin geschehen, so wird es von ihr mit Zuneigung belohnt. Somit aktiviert sie das Belohnungssystem des Kindes, welches den Missbrauch auf der Suche nach Nähe und Geborgenheit geschehen lässt. Im Zuge der starken emotionalen und sexuellen Verstrickung bittet sie das betroffene Kind, niemandem von dem Missbrauch zu erzählen. Sie stellt den Missbrauch beispielsweise als gemeinsames Geheimnis dar, das es zu hüten gilt. Sie verwirrt das Kind so in seiner Wahrnehmung von richtig und falsch, von übergriffig und nicht übergriffig, ,, sie sagte, das würden Mütter machen für ihre Söhne, und ich hätte ja keine mehr". Durch das Einführen in sexuelle Praktiken nimmt sie für die Betroffenen eine besondere Rolle ein. Ihr Missbrauch kann dazu führen, dass die Betroffenen von der parentifizierenden Täterin angeleitet, ihre Sexualität entdecken und sexuelle Gelüste entwickeln, welche die Täterin involvieren. Um diesen Vorgang zu decken, führt sie teilweise, wenn vorhanden, ein weiterhin aktives Sexualleben mit ihrem Partner, während die (sexuelle) Beziehung zum betroffenen Kind parallel dazu existiert.

\section{Die instruierende Täterin}

Die instruierende Täterin tritt im Kontext des sexuellen Kindesmissbrauchs in organisierten Gewaltstrukturen auf. Organisierte Gewalt bezeichnet einen Kontext, in dem ein Netzwerk von Tatpersonen Kinder und Jugendliche teilweise bis in ihr Erwachsenenalter hinein strategisch sexuell ausbeutet (Dr. Salter 2017). In diesem Kontext des Menschenhandels im Zusammenhang mit sexueller Ausbeutung besteht die Aufgabe der instruierenden Täterin darin, die betroffenen Kinder auf den sexuellen Missbrauch vorzubereiten. Es handelt sich um eine Art Training, da ein Handlungsablauf eingeübt wird, „die war da, um uns zu lehren. Dieses Lehren vom Sex hat sie uns beigebracht". Des Weiteren vermittelt sie den betroffenen Kindern Grundkenntnisse über Geschlechtsverkehr.

\section{Mischformen}

Die Ergebnisse haben gezeigt, dass der Täterinnentyp der sadistischen Täterin sowie der Typus der vermittelnden Täterin oftmals in einer Mischform aufzutreten scheinen, die verschiedene Charakteristika beider Typen vereint. Bei dieser Mischform führt die Täterin die betroffenen Kinder nicht nur Dritten zum sexuellen Missbrauch zu, sondern nimmt ebenfalls aktiv an diesem teil. In 5 von 8 Fällen ließ sich dieser Typus im Kontext der organisierten Gewalt finden. Die Übergabe des Kindes an weitere Täter:innen wird erzwungen, gleichzeitig wird jedoch auch das eigene Gewaltmotiv ausgelebt, „meine Mutter war sehr sadistisch, hat uns alleine gemacht die meiste Zeit. [...] hat uns wirklich gequält bis gefoltert. [...] wahrscheinlich hat die mich geboren, damit sie mich benutzen kann für solche Akte. Weil beim eigenen Kind fällt es am wenigsten auf. [...] Und ähm ... die haben ... meine Mutter hat sich sowieso ihr Taschengeld 
aufgebessert mit uns, ja“. Die Täterinnenstrategien zielen darauf $\mathrm{ab}$, betroffene Kinder zu vermitteln sowie ebenfalls aktiv missbrauchen zu können. Auch bei diesem Täterinnentypus ist eine Verschiebung der Schuld zu erkennen, sodass den Betroffenen die eigene Schuld am erlebten Missbrauch suggeriert wird. Durch Bedrohung sichert sich die Mischform der Typen das durch Angst erzeugte Schweigen der betroffenen Kinder und sichert sich zudem ab, indem sie den Missbrauch unter dem Konstrukt eines Vorwands durchführt. Dieser Vorwand ist eingebunden in das professionelle Handeln der Täter:innen, wie beispielsweise in spielerische Szenarien im Kindergartenalltag, „Prinzessinund-Ritter-Spiel und Stinki-Schweinchen-Spiel “.

Auch die instruierende Täterin scheint innerhalb dieser Strukturen in Verbindung mit der vermittelnden Täterin aufzutreten, sodass man von einer Vor- sowie Nachbereitung Betroffener sprechen kann. In diesem Falle lehrt die Täterin die betroffenen Kinder zunächst, wie sie sich im Falle des sexuellen Missbrauchs zu verhalten haben, um sie in einem nächsten Schritt den Täter:innen zuzuführen, ,ich erinnere mich daran, dass sie diejenige war, die uns immer hingebracht hat. Ich erinnere mich auch daran, dass sie [...] diejenige war, die mit uns geübt hat. Also die gesagt hat: Wenn es so und so ist, machst du das und das". Diese Mischform ließ sich ausschließlich innerhalb organisierter Gewaltstrukturen finden.

\section{Diskussion}

Das Ziel der Studie war es, die bisherige Forschung zu Täterinnentypologien im Bereich des sexuellen Kindesmissbrauchs um Ergebnisse aus Daten zu ergänzen, die im Kontext der UKASK erhoben wurden. Anhand einer qualitativen Inhaltsanalyse wurden typische Rollen der Täterin gegenüber dem betroffenen Kind sowie ihre Täterinnenstrategien herausgearbeitet.

11 der 30 analysierten Fälle stammen aus dem Kontext organisierter (ritueller) Gewaltstrukturen (Dr. Salter 2017; Dr. Schröder et al. 2020). Dies scheint den Unterschied der vorliegenden Täterinnentypologie zu in der vorherigen Forschung beschriebenen Täterinnentypologien zu begründen. Die vorliegende Täterinnentypologie ist daher als wichtige Ergänzung zu sehen. Die Betonung organisierter Gewaltstrukturen resultiert aus dem Ergebnis, dass die Typen der instruierenden Täterin sowie der sadistischen und vermittelnden Täterin in dem hier analysierten Material scheinbar insbesondere in diesen Strukturen häufig vorkommen. Hierbei ist wichtig zu erwähnen, dass in diesen Strukturen operierende Tatpersonen die von der Gewalt betroffenen Personen häufig durch Manipulation und Erpressung zur Mittäter:innenschaft zwingen (Behrendt et al. 2020).
Die Ergebnisse machen deutlich, dass die gewonnenen Kategorien die bisherigen Typologien von Täterinnen im Kontext des sexuellen Kindesmissbrauchs ergänzen: Die sadistische Täterin zeichnet sich durch ihr hohes Gewaltpotenzial aus. Zwar lässt sich dieser Typus innerhalb bestehender Typologien finden (beispielsweise in Enders 1995), oftmals werden derartige Handlungen jedoch als abweichend bzw. atypisch deklariert, was suggeriert, dass nur wenige Frauen diesem Typus zuzuordnen sind.

Auch Täterinnen im Bereich der organisierten Gewalt wurden bisher kaum innerhalb der Täterinnentypologien berücksichtigt. Drei der beschriebenen 4 Täterinnentypen der vorliegenden Typologie hingegen agieren zu einem gewissen Teil in diesen Strukturen und nehmen dort zentrale Rollen ein. Die vermittelnde Täterin erinnert an die „Madames“, welche nach Angaben von Amnesty International die sexuelle Ausbeutung von Kindern organisieren (Amnesty Journal 2019). Auch Europol veröffentlicht auf Europe's Most Wanted Fugitives immer wieder Steckbriefe von Frauen, die aktiv in den Menschenhandel involviert sind (Europol 2021). Zukünftige Studien sollten die Darstellung dieses Typus intensiver analysieren.

Frauen werden in gängigen Typologien oftmals als Mittäterinnen dargestellt (Matthews et al. 1991; Enders 1995; Saradjian und Hanks 1996; Prof. Dr. Kavemann und Braun 2002). In nur 2 der vorliegenden Fälle der Analyse fand der Missbrauch durch eine Frau sowie einen Mann in gemeinsamer Täterschaft statt. Hierbei nahm jedoch in beiden Fällen die Frau die führende Rolle ein. In diesem Zuge betonen Betroffene, deutlich mehr physische und psychische Gewalt durch die beteiligte Frau erfahren zu haben als durch den involvierten Mann. Ebenfalls wurde der Eindruck gewonnen, dass der Aspekt der von Frauen ausgehenden sexuellen Parentifizierung innerhalb der Fachliteratur nur sehr nebensächlich behandelt wird. Der Typus der parentifizierenden Täterin kommt am ehesten dem ,teacher/lover type“ von Matthews et al. (1991) nahe. Der Typ Täterin beschreibt eine Frau, die ihre Handlungen selbst als nicht kriminell, sondern den Betroffenen wohltuend ansieht. Die parentifizierende Täterin setzt ihren Missbrauch jedoch bewusst durch Befehle gegen den Willen des Kindes sowie durch eine aktive Instrumentalisierung der Mutterrolle um. Ob diesem Typus eine pädophile sexuelle Präferenz zugrunde liegt, konnte aufgrund der Determination durch subjektive Aussagen Betroffener nicht erschlossen werden.

Die vorliegende Studie und die damit einhergehende Täterinnentypologie können Hinweise für weitere, auch quantitative Forschung liefern. Eine zentrale Limitation der vorliegenden Täterinnentypologie ergibt sich aus der geringen Fallzahl, welche die Varianz der Datenbasis einschränkt. Zudem existiert die Möglichkeit, direkte Zitate $\mathrm{zu}$ veröffentlichen, nur eingeschränkt. Dies begrenzt die Augenscheinvalidität des Kategoriensystems im vorliegenden Be- 
richt. Die Fragestellung wurde Betroffenen und Zeug:innen im Rahmen der Interviews und Berichte nicht vorgegeben, sondern die Auswertung basiert auf einer subjektiven Interpretation. Täter:innentypologien sind zudem in sich limitiert, da sie anhand von Durchschnittsmerkmalen konstruiert werden und Idealtypen abbilden. Daher sind sie nicht vollständig auf Einzelfälle zurückzuführen.

\section{Schlussfolgerungen}

Die vorliegende Täterinnentypologie hat aufgezeigt, dass z.T. Täterinnen existieren, welche vermittelnd innerhalb organisierter Strukturen agieren, über ein großes Gewaltpotenzial verfügen sowie aus eigener Motivation missbrauchen. Diese Ergebnisse sollten für die Hypothesengenerierung in zukünftigen Studien zu Sexualstraftäterinnen genutzt werden. Es ist darüber hinaus wichtig, Aufklärungsarbeit zum Thema des sexuellen Kindesmissbrauchs durch Frauen zu leisten, um diese Fälle sichtbarer zu machen und präventive sowie interventionistische Strategien entwickeln zu können. Schulungen in pädagogischen Einrichtungen, der allgemeinen Gesundheitsversorgung, der Polizei sowie der Justiz würden zudem durch eine intensivere Aufklärung eine bessere Versorgung von einerseits Frauen mit sexuellem Interesse an Kindern, die ein Risiko für sexuelle Übergriffe auf Kinder aufweisen, und andererseits Betroffenen von sexuellem Kindesmissbrauch durch Frauen gewährleisten.

Danksagung Die Autor:innen danken allen Betroffenen und Zeug:innen im Kontext des sexuellen Kindesmissbrauchs durch eine Frau, die der UKASK ihre Berichte oder Anhörungen auch für die Forschung zur Verfügung gestellt haben.

Förderung Die vorliegende Studie wurde durch die Unabhängige Kommission zur Aufarbeitung sexuellen Kindesmissbrauchs (UKASK) gefördert, die vom Unabhängigen Beauftragten für Fragen des sexuellen Kindesmissbrauchs (UBSKM) ernannt wurde. Das Amt des UBSKM ist mit dem Bundesministerium für Familie, Senioren, Frauen und Jugend (BMFSJ) assoziiert, arbeitet jedoch unabhängig vom BMFSJ und ist nicht weisungsgebunden.

Funding Open Access funding enabled and organized by Projekt DEAL.

Interessenkonflikt P. Briken ist Mitglied der UKASK. T. Gebhardt, S. Tozdan und J. Schröder geben an, dass kein Interessenkonflikt besteht.

Open Access Dieser Artikel wird unter der Creative Commons Namensnennung 4.0 International Lizenz veröffentlicht, welche die Nutzung, Vervielfältigung, Bearbeitung, Verbreitung und Wiedergabe in jeglichem Medium und Format erlaubt, sofern Sie den/die ursprünglichen Autor(en) und die Quelle ordnungsgemäß nennen, einen Link zur Creative Commons Lizenz beifügen und angeben, ob Änderungen vorgenommen wurden.

Die in diesem Artikel enthaltenen Bilder und sonstiges Drittmaterial unterliegen ebenfalls der genannten Creative Commons Lizenz, sofern sich aus der Abbildungslegende nichts anderes ergibt. Sofern das betreffende Material nicht unter der genannten Creative Commons Lizenz steht und die betreffende Handlung nicht nach gesetzlichen Vorschriften erlaubt ist, ist für die oben aufgeführten Weiterverwendungen des Materials die Einwilligung des jeweiligen Rechteinhabers einzuholen.

Weitere Details zur Lizenz entnehmen Sie bitte der Lizenzinformation auf http://creativecommons.org/licenses/by/4.0/deed.de.

\section{Literatur}

Amnesty Journal (2019) Mafia, Madames und Mädchen. https:// www.amnesty.de/informieren/amnesty-journal/nigeria-mafiamadames-und-maedchen. Zugegriffen: 3. März 2021

Arzt S, Brunnauer C, Schartner B (2018) Sexualität, Macht und Gewalt. Anstöße für die sexualpädagogische Arbeit mit Kindern und Jugendlichen. Springer VS, Wiesbaden

ATLAS.ti Scientific Software Development GmbH (2018) ATLAS.ti 8 Windows - Quick Tour. http://downloads.atlasti.com/docs/ quicktour/QuickTour_a8_win_de.pdf. Zugegriffen: 12. Nov. 2020

Behrendt P, Schröder J, Nick S, Briken P (2020) Was ist sexualisierte Gewalt in organisierten und rituellen Strukturen? Eine qualitative Inhaltsanalyse der Erfahrungsberichte von Betroffenen. Z SexForsch 33(2):70-87. https://doi.org/10.1055/a-1160-3976

Bullens R (1995) Der Grooming-Prozess - oder das Planen des Missbrauchs. In: Marquard-Mau B (Hrsg) Schulische Prävention gegen sexuelle Kindesmisshandlung. Juventa, München

Denov MS (2003) The myth of innocence: Sexual scripts and the recognition of child sexual abuse by female perpetrators. J Sex Res 40(3):303-314

Enders U (1995) Vergiftete Kindheit - Frauen als Täterinnen. In: Auch Indianer kennen Schmerz - Sexuelle Gewalt gegen Jungen. Kiepenheuer \& Witsch, Köln, S 101-111

Europol (2021) Europe's most wanted fugitives. https://eumostwanted. eu/. Zugegriffen: 3. März 2021

Heiliger A (2001) Täterstrategien bei sexuellem Missbrauch und Ansätze der Prävention. In: beiträge zur feministischen theorie und praxis, S 71-82. http://www.anita-heiliger.de/htm/taeterstrategien_ bei_sexuellem_missbrauch.pdf. Zugegriffen: 8. Okt. 2020

Johansson-Love J, Fremouw W (2006) A critique of the female sexual perpetrator research. In: Aggression and violent behaviour, Bd. 11. Elsevier, Amsterdam, S 12-26

Kavemann B, Braun G (2002) Frauen als Täterinnen. In: Bange D, Körner W (Hrsg) Handwörterbuch Sexueller Missbrauch. Hogrefe, Göttingen, Bern, Toronto, Seattle, S 121-131

Matthews J, Mathews R, Speltz K (1991) Female sexual offenders: a typology. In: Patton M (Hrsg) Family sexual abuse: frontline research and evaluation. SAGE, Newbury Park, London, New Delhi

Mayring P (2010) Qualitative Inhaltsanalyse. Grundlagen und Techniken, 12. Aufl. Beltz, Weinheim Basel

Motz A (2014) The ultimate taboo? An exploration of female violence and perversion. Psychoanal Psychother 28(3):267-281

Niemeczek A (2014) Tatverhalten und Täterpersönlichkeit von Sexualdelinquenten: Der Zusammenhang von Verhaltensmerkmalen und personenbezogenen Eigenschaften. Springer VS, Wiesbaden

Salter M (2017) Organised sexual abuse. Routledge, New York

Sandler J, Freeman N (2007) Topology of Female Sex Offenders: A Test of Vandiver and Kercher. Sex Abuse 19(2):73-89

Saradjian J, Hanks H (1996) Women who sexually abuse children. From research to clinical practice. John Wiley \& Sons, Chichester, New York

Schröder J, Behrendt P, Nick S, Briken P (2020) Was erschwert die Aufdeckung organisierter und ritueller Gewaltstrukturen? Eine qualitative Inhaltsanalyse von Betroffenen und Zeitzeug_innen. In: Psychiatrische Praxis, Bd. 47. Thieme, Stuttgart, New York, S 249-259 
Syed F, Williams S (1996) Case studies of female sex offenders in the correctional service of Canada. Correctional Service of Canada, Ottowa

Tozdan S, Briken P, Dekker A (2019) Uncovering female child sexual offenders-needs and challenges for practice and research. JCM. https://doi.org/10.3390/jcm8030401
Unabhängige Kommission zur Aufarbeitung sexuellen Kindesmissbrauchs (2019) Bilanzbericht 2019. Unabhängige Kommission zur Aufarbeitung sexuellen Kindesmissbrauchs, Berlin 